\title{
An Introduction to The Irish Longitudinal Study on Ageing
}

\author{
Rose A. Kenny, MD, FRCPI, FRCP (Lond, Edin)
}

$T_{1}$ his supplement is designed to introduce the international health community to The Irish Longitudinal Study on Ageing (TILDA). ${ }^{1}$ TILDA is one of a family of longitudinal studies on aging that includes the Health and Retirement Survey (HRS; United States), ${ }^{2}$ the Survey of Health, Ageing and Retirement in Europe, ${ }^{3}$ The English Longitudinal Study of Ageing, ${ }^{4}$ the Longitudinal Aging Study in India, ${ }^{5}$ the China Health and Retirement Longitudinal Study, ${ }^{6}$ and the Japanese Study of Aging and Retirement, ${ }^{7}$ among others. The HRS has acted as a template for the design of these longitudinal aging studies around the world. Studies from the "HRS family" are designed to meet the needs of the populations they represent and to facilitate international comparisons. The studies share core elements such as the domains represented (health, social, and economic), specific questions, and methods of data collection. The studies may differ in the depth and type of additional information collected. TILDA has a particular emphasis on health and is unique in the extent of objective physical measurements made and use of health technologies.

The purpose of TILDA is to generate novel research to better understand the aging process and to inform national and international policy decisions on aging. In common with the HRS family of studies, the data are archived and available to all researchers. ${ }^{8}$ Eight thousand one hundred seventy-five community-dwelling persons aged 50 and older participated in Wave 1 and will participate in the study thereafter every 2 years. An additional 329 interviews were conducted with younger partners of eligible individuals. Proxy interviews (informants) are conducted during follow-up if participants are unable to take part because of physical or cognitive impairment or for end-of-life interviews. The response rate in Wave 1 was $62 \%$. Data were collected in home-based interviews for all respondents; additional information was collected

From The Irish Longitudinal Study on Ageing (TILDA), Trinity College, Dublin, Ireland.

Address correspondence to Professor Rose A. Kenny, The Irish Longitudinal Study on Ageing (TILDA), Trinity College, Dublin 2, Ireland. E-mail: rkenny@tcd.ie

DOI: $10.1111 /$ jgs. 12200 using self-completion postal questionnaires (response rate $84 \%$ ) and health assessments at a dedicated health center or in the home (response rate $72.3 \%$ ). Data were archived in December 2011. Wave 2 data collection was completed in December 2012 and will be archived in 2013.

The purpose of this supplement is to introduce the international community to TILDA, describe comparability with the "HRS family" of studies, and showcase the potential of TILDA - particularly for researchers in geriatric medicine. We hope to encourage the international research community to avail itself of this valuable data set.

The first article in the supplement ${ }^{9}$ sets out the main features of the design of the study, the main themes and topics it addresses, the sample design, fieldwork procedures used, response rates achieved, and weighting processes. The second article ${ }^{10}$ describes the chosen health assessments, details the rationale for selection of tests, and summarizes health assessment methodologies and technologies. This paper provides the framework for the third article, ${ }^{11}$ which describes population-based normative data from TILDA for a battery of physical and cognitive tests commonly used in the Comprehensive Geriatric Assessment: Mini-Mental State Examination, Montreal Cognitive Assessment, executive function measured using the Color Trails Test, height, weight, body mass index (BMI), bone density measured using heel bone ultrasound, Timed Up-and-Go (TUG) Test, walking speed, and grip strength. It is anticipated that readers will find this information and accompanying tables of use in day-to-day clinical practice. Another article ${ }^{12}$ describes the comparability of TILDA data with that of the HRS family and other studies and shows that the physical and cognitive health of older people in Ireland is closer to that of their English counterparts than of those in the United States and that similar health inequalities exist in all three countries. The final article ${ }^{13}$ examines the role of different life domains in determining quality of life (QoL) with comparisons with international results and illustrates that, even when physical health becomes frail, QoL can remain resilient as individuals find value and enjoyment in other dimensions of life.

Archived TILDA data in the United States can be accessed by making an application to the National Archive of Computerized Data on Aging. ${ }^{14}$ 


\section{REFERENCES}

1. Barrett A, Savva G, Timonen V et al. Fifty plus in Ireland 2011. First results from The Irish Longitudinal Study on Ageing. Dublin: The Irish Longitudinal Study on Ageing, 2011.

2. Juster FT, Suzman R. An overview of the Health and Retirement Study. J Hum Resour 1995;30:S7-S56.

3. Boersch-Supan A, Brugiavini A, Jurges A et al. Health Ageing and Retirement in Europe: First Results from the SHARE Survey. Mannheim, Germany: Mannheim Research Institute for the Economics of Ageing, 2005.

4. Marmot M, Banks J, Blundell R et al. Health, Wealth and Lifestyles of the Older Population in England: The 2002 English Longitudinal Study of Ageing. London: Institute for Fiscal Studies, 2002.

5. RAND Corporation, 2011. LASI'RAND Survey Meta Data Repository [on-line]. Available at https://mmicdata.rand.org/meta/?section=study\&studyid=36 Acessed February 26, 2013.

6. China Health and Retirement Longitudinal Study (CHARLS), 2011. HOME CHARLS [on-line]. Available at http://charls.ccer.edu.cn/en Accessed February 26, 2013

7. Research Institute of Economy, Trade and Industry (REITI), REITI - JSTAR (Japanese Study of Aging and Retirement) [on-line]. Available at http://www. rieti.go.jp/en/projects/jstar/index.html Accessed February 26, 2014.
8. Irish Social Science Data Archive (ISSDA), n.d. ISSDA The Irish Longitudinal Study on Ageing (TILDA). Available at http://www.ucd.ie/issda/data/ tilda/ Accessed February 26, 2013.

9. Whelan BJ, Savva GM. The design and methodology of The Irish Longitudinal Study on Ageing. J Am Geriatr Soc 2013;61:S265-S268.

10. Cronin H, O'Regan C, Kearney P et al. Health and aging: Development of The Irish Longitudinal Study on Ageing health assessment. J Am Geriatr Soc 2013;61:S269-S278.

11. Kenny RA, Coen RF, Frewen J et al. Normative values of cognitive and physical function in the older population: Findings from The Irish Longitudinal Study on Ageing. J Am Geriatr Soc 2013;61:S279-S290.

12. Savva GM, Maty SC, Setti A et al. Cognitive and physical health in the older populations of England, the United States, and Ireland: International comparability of The Irish Longitudinal Study on Ageing. J Am Geriatr Soc 2013;61:S291-S298.

13. Layte R, Sexton E, Savva G. Quality of life in older age: Evidence from an Irish cohort study. J Am Geriatr Soc 2013;61:S299-S305.

14. ICPSR/National Archive of Computerized Data on Aging, NCAD [on-line]. Available at http://www.icpsr.umich.edu/icpsrweb/NACDA/ Accessed February 26, 2013. 